\title{
Which 4-manifolds are toric varieties?
}

\author{
Stephan Fischli ${ }^{1}$, David Yavin ${ }^{2}$ \\ 1 Mathematisches Institut, Universität Bern, Sidlerstrasse 5, CH-3012 Bern, Switzerland \\ 2 Max-Planck Institut für Mathematik, Gottfried Claren Strasse 26, D-53225 Bonn, Germany
}

Received 19 August 1991; in final form 24 November 1992

\section{Definitions}

Let $\Sigma$ be a complete $d$-dimensional fan, i.e. a complex of closed convex polyhedral cones in $\mathbf{R}^{d}$ with apex 0 , generated by primitive lattice points $v_{1}, \ldots, v_{n} \in \mathbf{Z}^{d}$, such that $\bigcup_{\sigma \in \Sigma} \sigma=\mathbf{R}^{d}$. Denote the $q$-skeleton of $\Sigma$ by $\Sigma^{q}=\{\sigma \in \Sigma \mid \operatorname{dim} \sigma=q\}$. The polyhedral cells obtained by intersecting each cone $\sigma \in \Sigma, \sigma \neq\{0\}$, with the unit sphere $S^{d-1} \subset \mathbf{R}^{d}$ form a spherical complex $C$. Let $S(C)$ be the barycentric subdivision of $C$ and for $\sigma \in \Sigma, \sigma \neq\{0\}$, let $\hat{\sigma}$ be the union of all simplices of $S(C)$ whose vertices are barycenters of cells of $C$ which contain $\sigma \cap S^{d-1}$. For $\sigma=\{0\}$ we set $\hat{\sigma}=B^{d}$, the unit ball of $\mathbf{R}^{d}$, and call $\hat{\Sigma}=\{\hat{\sigma} \mid \sigma \in \Sigma\}$ the dual complex of $\Sigma$.

Let $T^{d}$ denote the $d$-dimensional torus $\mathbf{R}^{d} / \mathbf{Z}^{d}$. Each $k$-dimensional cone $\sigma \in \sum$ spans a $k$-dimensional subspace of $\mathbf{R}^{d}$ which, since it has a rational basis, maps under the projection $\mathbf{R}^{d} \rightarrow T^{d}$ to a $k$-dimensional subtorus $T_{\sigma} \subseteq T^{d}$. The toric variety $X_{\Sigma}$ is defined to be the quotient $B^{d} \times T^{d} / \sim$ where $(x, t) \sim\left(x^{\prime}, t^{\prime}\right)$ if and only if $x=x^{\prime} \in$ int $\hat{\sigma}$ and $t$ and $t^{\prime}$ belong to the same orbit of the natural action of $T_{\sigma}$ on $T^{d}$. Note that the torus $T^{d}$ over a point $x \in$ int $\hat{\sigma}$ is collapsed by the relation $\sim$ to the quotient $\hat{T}_{\sigma}=T^{d} / T_{\sigma}$ which is a torus of dimension $d-\operatorname{dim} \sigma=\operatorname{dim} \hat{\sigma}$.

We denote by $p$ the obvious projection of $X_{\Sigma}$ onto $B^{d}$ and will identify $p^{-1}$ (int $\hat{\sigma}$ ) with int $\hat{\sigma} \times \hat{T}_{\sigma}$.

The proofs of the following properties can be found e.g. in [2].

(i) The toric variety $X_{\Sigma}$ is compact and simply-connected (since $\Sigma$ is complete).

(ii) The toric variety $X_{\Sigma}$ is a smooth manifold if and only if $\Sigma$ is a simplicial fan and $\left|\operatorname{det}\left(v_{1}, \ldots, v_{d}\right)\right|=1$ whenever $v_{1}, \ldots, v_{d} \in \mathbf{Z}^{d}$ are the primitive spanning vectors of a $d$-dimensional cone in $\Sigma$.

(iii) If $\Sigma$ and $\Sigma^{\prime}$ are two $d$-dimensional fans, and if there exists a unimodular transformation of $\mathbf{R}^{d}$ which maps the generating vectors of $\Sigma$ onto the generating vectors of $\Sigma^{\prime}$ and which induces a combinatorial isomorphism between $\Sigma$ and $\Sigma^{\prime}$, then the associated toric varieties $X_{\Sigma}$ and $X_{\Sigma^{\prime}}$ are homeomorphic in general, and diffeomorphic when either (hence the other) is smooth. 

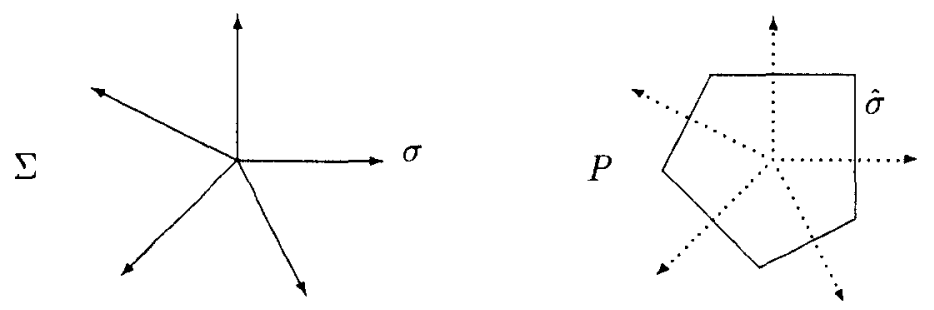

Fig. 1. A 2-dimensional fan and its dual complex

\section{CW-cell decomposition and cellular homology}

In the following we restrict our attention to a 4-dimensional toric variety $X=X_{\Sigma}$. Since the underlying fan $\Sigma$ is 2-dimensional, its dual complex $\hat{\Sigma}$ may be represented as the face complex of a polygon $P$ with $0 \in$ int $P$ and such that each edge of $P$ is perpendicular to its dual 1-cone (see Fig. 1).

We first describe a CW-cell decomposition of $X$ which arises from the face structure of $P$ and the standard CW-cell decomposition of the 2-dimensional torus $T^{2}$. The latter is given by the cells $\tau_{0}, \tau_{1}, \tau_{2}$ and $\tau_{12}$ which are represented, respectively, by the subsets $\{(0,0)\},] 0,1[\times\{0\},\{0\} \times] 0,1[$ and $] 0,1[\times] 0,1\left[\right.$ of $\mathbf{R}^{2}$. By suitably embedding the tori $T^{0}$ and $T^{1}$ into $T^{2}$, the subcomplexes $\left\{\tau_{0}\right\}$ and $\left\{\tau_{0}, \tau_{1}\right\}$ can be regarded as cell decompositions of $T^{0}$ and $T^{1}$, respectively. Note that the closures of these cells represent cycles in the cellular homology of the corresponding tori.

In order to obtain a cell decomposition of the torus $\hat{T}_{\sigma}$ which lies over each of the interior points of the face $\hat{\sigma}$ of $P$, we use an explicit homeomorphism $h_{\sigma}: \hat{T}_{\sigma} \rightarrow T^{2-\operatorname{dim} \sigma}$. For a 1-dimensional cone $\sigma \in \Sigma$ generated by the vector $v_{\sigma} \in \mathbf{Z}^{2}$, $h_{\sigma}$ is induced by the projection $g_{\sigma}: \mathbf{R}^{2} \rightarrow \mathbf{R}$ which maps $x \in \mathbf{R}^{2}$ to $\operatorname{det}\left(v_{\sigma}, x\right)$. For a 2-dimensional cone $\sigma$ we set $h_{\sigma}=0$ and for $\sigma=\{0\}, h_{\sigma}=\mathrm{id}_{T^{2}}$.

For each cone $\sigma$ and each cell $\tau$ in $T^{2-\operatorname{dim} \sigma}$ we define the cell

$$
c_{\sigma, t}=\operatorname{int} \hat{\sigma} \times h_{\sigma}^{-1}(\tau)
$$

Note that $\operatorname{dim} c_{\sigma, \tau}=\operatorname{codim} \sigma+\operatorname{dim} \tau$. The cells $c_{\sigma, \tau}$ form a CW-decomposition of $X$, and if we provide them with appropriate orientations, then the boundaries of the corresponding cellular chains are given by

$$
\begin{aligned}
\sigma \in \Sigma^{2}: & \partial_{0} c_{\sigma, \tau_{0}}=0 \\
\sigma \in \Sigma^{1}: & \partial_{1} c_{\sigma, \tau_{0}}=c_{\sigma^{\prime}, \tau_{0}}-c_{\sigma^{\prime \prime}, \tau_{0}} \\
\partial_{2} c_{\sigma, \tau_{1}} & =0 \\
\partial_{2} c_{\sigma, \tau_{0}} & =\sum c_{Q, \tau_{0}} \\
\partial_{3} c_{\sigma, \tau_{1}} & =\sum\left(-v_{Q}^{2}\right) c_{Q, \tau_{1}} \\
\partial_{3} c_{\sigma, \tau_{2}} & =\sum v_{Q}^{1} c_{Q, \tau_{1}} \\
\partial_{4} c_{\sigma, \tau_{12}} & =0
\end{aligned}
$$


where $\sigma^{\prime}, \sigma^{\prime \prime}$ are the unique 2-dimensional cones which contain $\sigma \in \Sigma^{1}, \varrho$ ranges over $\Sigma^{1}$ in all the sums, and $v_{Q}^{1}, v_{Q}^{2}$ are the coordinates of the primitive vector $v_{Q}$ which generates the cone $\varrho$. The multiplicities of $c_{Q, \tau_{1}}$ in the boundaries of the two 3-dimensional chains are the images of the vectors $(1,0)$ and $(0,1)$ under the projection $g_{\varrho}: \mathbf{R}^{2} \rightarrow \mathbf{R}$ described above.

Thus we obtain the following homology groups of $X$ :

$$
H_{q}(X) \cong \begin{cases}\mathbf{Z} & q=0,4 \\ \mathbf{Z}^{n} /\left(\mathbf{Z} v_{1}^{*}+\mathbf{Z} v_{2}^{*}\right) \cong \mathbf{Z}^{n-2} \oplus \mathbf{Z} / m \mathbf{Z} & q=2 \\ 0 & \text { otherwise }\end{cases}
$$

where $n=\operatorname{card} \Sigma^{1}, v_{1}^{*}$ (resp. $\left.v_{2}^{*}\right) \in \mathbf{Z}^{n}$ is the vector whose components are the first (resp. second) coordinates of the vectors $v_{\sigma}, \sigma \in \Sigma^{1}$, and $m$ is the greatest common divisor of the determinants $\operatorname{det}\left(v_{\sigma}, v_{\sigma^{\prime}}\right), \sigma, \sigma^{\prime} \in \Sigma^{1}$. In particular $H_{2}(X) \cong \mathbf{Z}^{n-2}$ if $X$ is smooth.

\section{The intersection form}

In this section we calculate the intersection form of a smooth 4-dimensional toric variety $X=X_{\Sigma} \cdot{ }^{1}$ Let $\sigma_{1}, \ldots, \sigma_{n}$ be the 1-dimensional cones of $\Sigma$, numbered counterclockwise, and for each $i$ let $v_{i}=\left(v_{i}^{1}, v_{i}^{2}\right) \in \mathbf{Z}^{2}$ be the primitive vector generating $\sigma_{i}$. We will assume from here on that the indexing from 1 to $n$ is cyclic so that any index which falls below 1 (resp. above $n$ ) has $n$ added to it (resp. subtracted from it). By property (ii) we have

$$
\operatorname{det}\left(v_{i}, v_{i+1}\right)=1 \quad(i=1, \ldots, n),
$$

and in view of property (iii) we may assume in addition that $v_{n-1}=(1,0)$ and $v_{n}=(0,1)$.

By (1) the group of 2-cycles of $X$ is generated by the chains $c_{\sigma_{i}, \tau_{1}}, i=1, \ldots, n$, and the corresponding homology classes $z_{i} \in H_{2}(X)$ satisfy the following relations induced by the boundaries of the two 3-chains:

$$
\begin{array}{r}
v_{1}^{1} z_{1}+\cdots+v_{n-2}^{1} z_{n-2}+z_{n-1}=0 \\
v_{1}^{2} z_{1}+\cdots+v_{n-2}^{2} z_{n-2}+z_{n}=0 .
\end{array}
$$

In order to determine the intersection numbers $z_{i} \cdot z_{j}$, we first observe that the spheres $p^{-1}\left(\hat{\sigma}_{i}\right)$ and $p^{-1}\left(\hat{\sigma}_{j}\right)$, which represent the classes $z_{i}$ and $z_{j}$ respectively, do not intersect in $X$ if the cones $\sigma_{i}$ and $\sigma_{j}$ are not adjacent in $\Sigma$. Therefore

$$
z_{i} \cdot z_{j}=0 \quad(1<|i-j|<n-1) .
$$

Second, the spheres $p^{-1}\left(\hat{\sigma}_{i}\right)$ and $p^{-1}\left(\hat{\sigma}_{i+1}\right)$ intersect in the unique point which lies over the vertex $\hat{\sigma}_{i} \cap \hat{\sigma}_{i+1}$ of $P$. Since $\operatorname{det}\left(v_{i}, v_{i+1}\right)=1$, it can be seen that this intersection is transversal and hence

$$
z_{i} \cdot z_{i+1}= \pm 1 \quad(i=1, \ldots, n)
$$

\footnotetext{
${ }^{1}$ This could be done by using the algebraic description of the cohomology ring of $X$ given by Danilov (see [2, Theorem 10.8]). However, we compute the actual intersection numbers of the generating 2-cycles using our cell decomposition of $X$
} 
where the signs are all equal and only depend on the orientation of $X$. In the following we fix them to be +1 .

Third, by multiplying both relations (3) with $z_{i}$ and taking a suitable linear combination of the resulting equations, we obtain the self intersection numbers

$$
d_{i}:=z_{i} \cdot z_{i}=-\operatorname{det}\left(v_{i-1}, v_{i+1}\right) \quad(i=1, \ldots, n)
$$

where we have also used the smoothness condition (2).

\section{Characterisation of the intersection form}

Having calculated the intersection form of $X$, we now characterise it up to equivalence, i.e. up to a change of basis of $\mathrm{H}_{2}(X)$. (For a treatment of symmetric bilinear forms in general see [8].) By (3) the classes $z_{1}, \ldots, z_{n-2}$ form a basis of $\mathrm{H}_{2}(\mathrm{X})$, and since by Poincare duality the intersection form is always non-singular, its rank equals $n-2$.

In order to determine the signature, we first calculate the principal minors $D_{k}=\operatorname{det}\left(\left(z_{i} \cdot z_{j}\right)_{1 \leqq i, j \leqq k}\right)$ of the intersection matrix. (Henceforth we will not distinguish between the intersection form and its matrix.) By the results of the previous section we have $D_{1}=d_{1}$ and

$$
D_{k}=d_{k} D_{k-1}-D_{k-2} \quad(k=2, \ldots, n-2),
$$

where we have set $D_{0}=1$. By induction one can easily prove, e.g. by using the Grassmann-Plücker relation in $\mathbf{R}^{2}$, that

$$
D_{k}=(-1)^{k+1} \operatorname{det}\left(v_{k+1}, v_{n}\right) \quad(k=1, \ldots, n-2) .
$$

From this equation we see that if none of the vectors $v_{k}$ is equal to $-v_{n}=(0,-1)$, then all the principal minors are non-zero and have alternating signs, except for the unique pair $\left(D_{k-1}, D_{k}\right)$ for which the vectors $v_{k}$ and $v_{k+1}$ lie on opposite sides of the $y$-axis. Hence by Jacobi's theorem the signature of the intersection form equals $4-n$. By a rule of Gundenfinger this still holds even if their exists a vector $v_{k}=(0,-1)$ in which case $D_{k-1}=0$ (see [5, note 1 on p. 304]).

If $n=3$ then there is only one possible vector $v_{1}=(-1,-1)$ and the intersection form of $X$ given by the matrix $(+1)$ is positive definite. If $n>3$ then the absolute value of the signature is less than the rank, hence the form is indefinite and can therefore be characterised by finally determining its type.

The even type is only possible if $n=4 .^{2}$ Indeed, if the intersection form of $X$ is even, then all of the self intersection numbers $d_{i}$ must be even. Since the vectors $v_{i}$ are primitive, it follows that each must have one even and one odd coordinate, thus they are contained in the lattice $\Gamma=(1,0)+\mathbf{Z}(1,1)+\mathbf{Z}(-1,1)$. Hence by Pick's formula (see e.g. [7]) the area $A(S)$ of the star-shaped polygon $S=\bigcup_{i=1}^{n} \operatorname{conv}\left\{0, v_{i}, v_{i+1}\right\}$ is given by

$$
\frac{A(S)}{\operatorname{det} \Gamma}=\operatorname{card}(\Gamma \cap \operatorname{int} S)+\frac{1}{2} \operatorname{card}(\Gamma \cap \partial S)-1
$$

\footnotetext{
${ }^{2}$ This also follows from a theorem of Donaldson (see [3, Theorem B]) which says that if the intersection form of a smooth simply-connected 4-manifold is indefinite and even and if the absolute value of its signature is 2 less than its rank, then its rank equals 2
} 

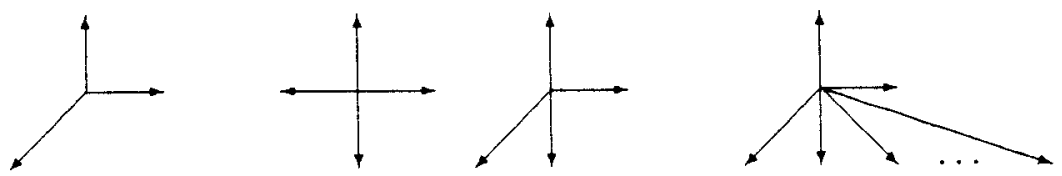

Fig. 2. Representative fans with 3,4 and $n$ generators

where $\operatorname{det} \Gamma$ denotes the determinant of a basis of $\Gamma$ and in our case is equal to 2 . But by condition (2) the area $A(S)$ equals $n / 2$ and $S$ does not contain any points of $\Gamma$ other than its vertices. Therefore the equality can hold only if $n=4$.

In fact, if $n=4$ and $v_{1}=(-1,0), v_{2}=(0,-1)$ the resulting form is even. On the other hand, every odd indefinite intersection form of rank $n \geqq 4$ can also be realised, e.g. by setting $v_{1}=(i-2,-1), i=1, \ldots, n-2$.

Thus we have completely characterised the possible intersection forms of $X$ (Fig. 2 shows the corresponding fans), and we summarize the results in the following

Theorem. $A$ non-singular integral symmetric bilinear form $B$ can be realised as the intersection form of an oriented smooth 4-dimensional toric variety $X$ if and only if either

(i) $\operatorname{rank}(B)=1$ and $B=( \pm 1)$, or

(ii) $\operatorname{rank}(B)=2$ and $B$ is even, or

(iii) $\operatorname{rank}(B) \geqq 2,|\operatorname{signature}(B)|=\operatorname{rank}(B)-2$ and $B$ is of odd type.

\section{Topological classification}

In 1982 Freedman characterised topological 4-manifolds and showed that every non-singular integral symmetric bilinear form can be realised as the intersection form of an oriented closed simply-connected 4-manifold. Moreover any two such manifolds realising the same form are homeomorphic if the form is even, whereas if the form is odd there are two homeomorphism classes, one with trivial and the other with non-trivial Kirby-Siebenmann obstruction (see [4, Theorem 1.5]).

In our case it is easy to give representatives of 4-manifolds which realise the intersection forms described in the theorem of the previous section. Namely, let us consider the oriented complex projective plane $\mathbf{C} P^{2}$ which has intersection form $(+1)$. Then $\mathbf{C} P^{2}$ with the opposite orientation, which we denote by $-\mathbf{C} P^{2}$, has intersection form $(-1)$. Furthermore, if we take the connected sum of $\mathbf{C} P^{2}$ with a finite number of copies of $-\mathbf{C P} P^{2}$, we obtain a 4-manifold whose intersection form is the orthogonal sum of $(+1)$ with a finite number of $(-1)$ and hence satisfies condition (iii) of the theorem. Finally, the even indefinite form of rank 2 is the intersection form of the product of spheres $S^{2} \times S^{2}$. All these manifolds are differentiable and hence have trivial Kirby-Siebenmann obstruction, and since the same is true for the toric varieties in question, we can state the following

Corollary. The homeomorphism classes of smooth 4-dimensional toric varieties are represented by the complex projective plane $\mathrm{CP} P^{2}$, the product of spheres $S^{2} \times S^{2}$ and the connected sum of $\mathbf{C} P^{2}$ with a finite number of copies of $-\mathbf{C P}$. 


\section{$6 C^{\infty}$-classification}

We now describe a handlebody decomposition of a smooth 4-dimensional toric variety $X$, and using elementary Kirby moves show that the smooth manifolds listed in the previous section in fact represent the different diffeomorphism classes of smooth 4-dimensional toric varieties (see [6] for a general reference on Kirby calculus). We use the same conventions on indices as in Sect. 3.

For any cone $\sigma \in \Sigma$, set $X_{\sigma}=p^{-1}(\sigma \cap P)$ (recall Fig. 1). When $\sigma$ is the 0 dimensional cone, $X_{\sigma}=T^{2}$. For each of the 1 -cones $\sigma_{i}, 1 \leqq i \leqq n, X_{\sigma}$ is a solid torus. Because of our smoothness hypothesis, for adjacent 1 -cones $\sigma_{i}$ and $\sigma_{i+1}, X_{\sigma_{i}} \cup X_{\sigma_{i+1}}$ is a 3-sphere with a canonical Heegaard splitting as two solid tori glued together along their bounding 2-tori. Denote by $\sigma_{i, i+1}$ the 2-cone bounded by $\sigma_{i}$ and $\sigma_{i+1} . X_{\sigma_{i, i+1}}$ is a 4 -ball $D^{4}$ bounded by the 3 -sphere $X_{\sigma_{i}} \cup X_{\sigma_{i+1}}$ and centered at the point $p^{-1}\left(\hat{\sigma}_{i, i+1}\right)$. It has a canonical product structure $D^{2} \times D^{2}$ such that $X_{\sigma_{i}}=\partial D^{2} \times D^{2}$ and $X_{\sigma_{i+1}}=D^{2} \times \partial D^{2}$. Thus for each $i, X_{\sigma_{i, i+1}}$ can be considered canonically as a 0 -handle, 2 -handle or 4 -handle and by arbitrarily designating a 0-handle and a 4-handle (in fact we choose $X_{\sigma_{n-1, n}}$ as the 4-handle), we obtain a smooth handlebody decomposition of $X$ with a unique 0-handle, a unique 4-handle, $n-2$ 2-handles and no 1- or 3-handles.

To obtain the correct Kirby calculus picture, note that the union of two adjacent handles $X_{\sigma_{i-1, i}} \cup X_{\sigma_{i, i+1}}$ is a disk bundle over the 2-sphere $p^{-1}\left(\hat{\sigma}_{i}\right)$ (with Euler number $d_{i}$ ), and two such adjacent disk bundles are glued together over a common $D^{2} \times D^{2}$ by reversing the order of the coordinates. Thus when the interior of the 4-handle is removed, the punctured toric variety $\tilde{X}:=X \backslash$ int $D^{4}$ is a plumbing on the graph with $n-2$ vertices shown in Fig. 3(a). The corresponding Kirby calculus picture for $X$ is shown in Fig. 3(b). As usual, the linking matrix is just the intersection matrix of $X$ so that the framings are the self intersection numbers $d_{1}, \ldots, d_{n-2}$ calculated in Sect. 3 .

Since the boundary of $\tilde{X}$ is a 3 -sphere, we could apply a theorem of Akbulut and Kirby [1, Corollary 4.2] to complete the proof (the theorem of Akbulut and Kirby is more general and only requires that the boundary be a homotopy 3-sphere), but in our setting we are able to give the following more direct argument.

By Poincaré duality, the intersection matrix $B$ calculated in Sect. 3 is unimodular. It follows by straightforward row reduction (over $\mathbf{Q}$ ) that one of the diagonal entries $d_{i}$ must satisfy $\left|d_{i}\right| \leqq 1$. Thus with elementary Kirby moves, we can split off from the picture a manifold which is either $\mathbf{C} P^{2}$ (if $\left.d_{i}=1\right),-\mathbf{C} P^{2}$ (if $d_{i}=-1$ ) or an $S^{2}$-bundle over $S^{2}$ (if $d_{i}=0$ ). Up to diffeomorphism there are only two such bundles, namely the trivial bundle $S^{2} \times S^{2}$ and $C P^{2} \#\left(-C P^{2}\right)$. If we have blown down a $\pm \mathbf{C P} P^{2}$, the remaining framed link diagram is obtained from the original

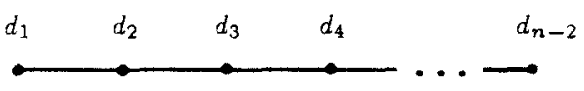

(a)

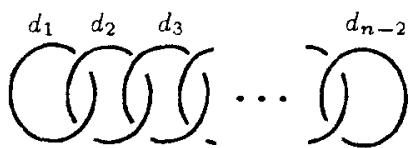

(b)

Fig. 3. a The plumbing diagram for $\tilde{X}$. b The framed link picture for $X$ 
one by erasing the $i^{\text {th }}$ component, linking the two adjacent ones to each other (unless $i=1$ or $i=n-2$ ), and subtracting $d_{i}$ from the framings of each. When $d_{i}=0$, the $i^{\text {th }}$ component is erased and the two adjacent ones are merged to a single link component with framing $d_{i-1}+d_{i+1}$ (unless $i=1$ or $i=n-2$, in which case the $i^{\text {th }}$ component and its adjacent component are simply erased from the diagram). In each of these cases, the framed link diagram which remains, if it is not empty, is of the same form as in Fig. 3(b) and still represents a closed manifold. Thus the linking matrix of the reduced diagram is necessarily unimodular, has the same form as $B$ and has lower rank.

It follows by induction that $X$ is diffeomorphic to a connected sum of the form $\stackrel{k}{\#} \mathrm{C} P^{2} \stackrel{l}{\#}\left(-\mathrm{C} P^{2}\right) \stackrel{m}{\#} S^{2} \times S^{2}$. But by the results of the previous section $b_{2}^{+}=1$ whence either $k$ or $m$ must equal 0 and the other must equal 1. Finally, since $S^{2} \times S^{2} \#\left(-\mathbf{C} P^{2}\right) \cong \mathbf{C} P^{2} \#\left(-\mathbf{C} P^{2}\right) \#\left(-\mathbf{C} P^{2}\right)$ we obtain

Theorem. The diffeomorphism classes of smooth 4-dimensional toric varieties are represented by the complex projective plane $\mathbf{C} P^{2}$, the product of spheres $S^{2} \times S^{2}$ and the connected sum of $\mathbf{C} P^{2}$ with a finite number of copies of $-\mathbf{C P}$.

Remarks. (i) If for some $i,\left|\operatorname{det}\left(\sigma_{i}, \sigma_{i+1}\right)\right| \neq 1$ then $X_{\sigma_{i}} \cup X_{\sigma_{i+1}}$ is a lens space which is not a sphere. Thus in general the sets $X_{\sigma_{i, i+1}}$ are cones on lens spaces and are attached to each other just as in the smooth case by gluing one solid torus to another. The authors do not know of an analogue of Kirby calculus which applies to such a space.

(ii) In any dimension $d$ we can define the sets $X_{\sigma}=p^{-1}\left(\sigma \cap B^{d}\right)$ for each $\sigma \in \Sigma$. If $\sigma$ is a $d$-dimensional cone, then $X_{\sigma}$ is a cone neighborhood of the point $p^{-1}(\hat{\sigma})$, and when $X$ is smooth it is a $2 d$-ball with a canonical product structure $D^{2} \times \cdots \times D^{2}$. This again gives rise to a handlebody decomposition (with no odd-dimensional handles) when $X$ is smooth.

Acknowledgements. Special thanks to Bob Gompf. The analysis of the Kirby link in Sect. 6 is largely due to him.

\section{References}

1. Akbulut, S., Kirby, R.: Branched Covers of Surfaces in 4-Manifolds. Math. Ann. 252, 111-131 (1980)

2. Danilov, V.I.: The Geometry of Toric Varieties. Russ. Math. Surv. 33, 97-154 (1978)

3. Donaldson, S.K.: Connections, Cohomology and the Intersection Forms of 4-Manifolds. J. Differ. Geom. 24, 275-341 (1986)

4. Freedman, M.H.: The Topology of 4-Dimensional Manifolds. J. Differ. Geom. 17, 357-453 (1982)

5. Gantmacher, F.R.: The Theory of Matrices, Vol. 1. New York: Chelsea 1959

6. Kirby, R.C.: The Topology of 4-Manifolds. (Lect. Notes Math., vol. 1374) Berlin Heidelberg New York: Springer 1989

7. Macdonald, I.G.: The Volume of a Lattice Polyhedron. Proc. Camb. Philos. Soc. 59, 719-726 (1963)

8. Milnor, J., Husemoller, D.: Symmetric Bilinear Forms. Berlin Heidelberg New York: Springer 1973 